\title{
THERMALLY STIMULATED DEPOLARIZATION CURRENT OF TWO-COMPONENT HETEROGENEOUS SOLID
}

\author{
J. PoŹNIAK \\ Institute of Low Temperature and Structure Research, Polish Academy of Sciences \\ P.O. Box 937, 50-950 Wrocław, Poland
}

(Received April 26, 1994)

\begin{abstract}
A simplified theory of thermally stimulated depolarization current of a parallel-plate condenser filled with heterogeneous solid consisting of two dielectrics is presented. It is assumed that the particles (with different shapes and dimensions) of one dielectric are sparsely distributed in another dielectric. A second basic assumption is that the average field in the solid is equal to the external electric field.
\end{abstract}

PACS numbers: 77.22.Ej

\section{Introduction}

Various theories have been elaborated and successfully applied to describe the relaxation effects induced by the action of an external alternating field on some heterogeneous systems (e.g. [1-3]). It should be noticed, however, that characteristic material parameters like conductivity and dielectric permittivity, appearing in pertinent relations, are treated as constants, and consequently these theories can be applied to describe the isothermal effects only. Theoretical approach to the relaxation processes appearing at changing temperature, like thermally stimulated polarization (TSP) and depolarization (TSD) currents, was presented in Refs. [4-10], in which the material parameters are treated as temperature dependent variables. It has been also shown that under non-isothermal conditions the application of equivalency concept in the case of elementary circuits of Maxwell and Wagner-Voigt type is drastically restricted $[11,12]$.

Simplified theories of TPS and TSD currents in heterogeneous dielectric systems, in which the charge carriers are accumulated at the interfaces of the components, have been proposed by Harašta and Thurzo [13], and by van Turnhout [4]. These theories are based on the postulate of two-layer series model with well-defined thicknesses of the layers. 
In the present paper an attempt is made to perform calculations of the TSD current of a parallel-plate condenser filled with the heterogeneous solid. It is assumed that the particles (with different shapes and dimensions) of one dielectric are sparsely distributed in another dielectric.

\section{Model assumptions and analytical solutions}

Let the two substances have different dielectric constants $\left(\varepsilon_{1}\right.$ and $\left.\varepsilon_{2}\right)$ as well as conductivities $\left(\kappa_{1}\right.$ and $\left.\kappa_{2}\right)$ and occupy fractions $v_{1}$ and $v_{2}$ of the total volume $v=1$ (i.e. the volume between the plates of the condenser), i.e.

$$
v_{1}+v_{2}=1 \text {. }
$$

Consider now the electric field $E$ in the heterogeneous solid. Its value averaged over the total volume $v$ is

$$
\bar{E}=\int_{v} \frac{E \mathrm{~d} v}{v} .
$$

For the component with the dielectric constant $\varepsilon_{1}$ and volume $v_{1}$, the average value of $E$ is

$$
\bar{E}_{1}=\int_{v_{1}} \frac{E \mathrm{~d} v}{v_{1}}
$$

and, similarly, for the second component

$$
\bar{E}_{2}=\int_{v_{2}} \frac{E \mathrm{~d} v}{v_{2}} .
$$

Therefore

$$
E=\frac{1}{V} \int_{V} E \mathrm{~d} v=\frac{1}{V}\left[\int_{v_{1}} E \mathrm{~d} v+\int_{v_{2}} E \mathrm{~d} v\right]=v_{1} \bar{E}_{1}+v_{2} \bar{E}_{2} .
$$

Now, we assume that the system is subject to the action of external static electric field $\bar{E}=U_{\mathrm{p}} / d$ at a temperature $T_{\mathrm{p}}$, where $U_{\mathrm{p}}$ is the applied voltage and $d$ is the distance between electrodes (this assumption is valid if the electrodes are not blocking). Then, from Eq. (2), we obtain

$$
U_{\mathrm{p}} / d=v_{1} \bar{E}_{1}+v_{2} \bar{E}_{2} \text {. }
$$

Under the action of the external electric field a surface charge $q(t)$ will be accumulated at the boundaries between the neighbouring phases. The continuity equation yields for the increase in the charge density

$$
\frac{\mathrm{d} q(t)}{\mathrm{d} t}=\kappa_{1} \bar{E}_{1}-\kappa_{2} \bar{E}_{2},
$$

the increase that is determined by the difference between ohmic conduction currents. Moreover, for the relation between the electric fields and $q(t)$ we have from the Gauss law

$$
q(t)=\varepsilon_{0} \varepsilon_{1} \bar{E}_{1}-\varepsilon_{0} \varepsilon_{2} \bar{E}_{2},
$$

where $\varepsilon_{0}$ is the permittivity of the free space. 
The spatial continuity of two current densities within two substances yields

$$
\varepsilon_{0} \varepsilon_{1} \frac{\mathrm{d} \bar{E}_{1}}{\mathrm{~d} t}+\kappa_{1} \bar{E}_{1}=\varepsilon_{0} \varepsilon_{2} \frac{\mathrm{d} \bar{E}_{2}}{\mathrm{~d} t}+\kappa_{2} \bar{E}_{2} \text {. }
$$

Substitution of $\bar{E}_{2}$ from Eq. (3) into the last equation yields

$$
\frac{\mathrm{d} \bar{E}_{1}}{\mathrm{~d} t}+\frac{1}{\tau} \bar{E}_{1}=\frac{\kappa_{2} U_{\mathrm{p}}}{d \varepsilon_{0}\left(\varepsilon_{1} v_{2}+\varepsilon_{2} v_{1}\right)}
$$

where

$$
\tau^{-1}=\frac{\kappa_{1} v_{2}+\kappa_{2} v_{1}}{\varepsilon_{0}\left(\varepsilon_{1} v_{2}+\varepsilon_{2} v_{1}\right)}
$$

The solution of Eq. (7) is

$$
\bar{E}_{1}=\left[\bar{E}_{1}(0)-\bar{E}_{1}(\infty)\right] \mathrm{e}^{-t / \tau}+\bar{E}_{1}(\infty),
$$

where $\bar{E}_{1}(0)=\left.\bar{E}_{1}(t)\right|_{t=0}$ and $\bar{E}_{1}(\infty)$ denotes the final value of $\bar{E}_{1}$ (i.e. at $t \rightarrow \infty$ ).

The initial value $\bar{E}_{1}(0)$ can be found from Eq. (2) and Eq. (5) by taking into account that the initial value of charge density, $q(0)$, is equal to zero. Consequently we obtain that

$$
\bar{E}_{1}(0)=E_{\mathrm{p}} \frac{\varepsilon_{2}}{\varepsilon_{1} v_{2}+\varepsilon_{2} v_{1}},
$$

where $E_{\mathrm{p}}=U_{\mathrm{p}} / d$.

Equation (4) makes it clear that the interfacial charge $q(t)$ reaches its maximum value when the ohmic currents become equal, i.e.

$$
\kappa_{1} E_{1}(\infty)=\kappa_{2} E_{2}(\infty)
$$

Introduction of $\bar{E}_{2}(\infty)$ from Eq. (3) into this relation yields

$$
\bar{E}_{1}(\infty)=E_{\mathrm{p}} \frac{\kappa_{2}}{\kappa_{1} v_{2}+\kappa_{2} v_{1}} \text {. }
$$

In measurements of TSD currents we are mainly interested in the interfacial charge, because it is this charge that can be frozen in. In agreement with Eq. (10) we can obtain that

$$
\bar{E}_{1}(0)=\frac{\varepsilon_{1} v_{2}+\varepsilon_{2} v_{1}}{v_{2}}=E_{\mathrm{p}} \frac{\varepsilon_{2}}{v_{2}} .
$$

For.this reason Eq. (5) becomes

$$
q(t)=\varepsilon_{0} \frac{\varepsilon_{1} v_{2}+\varepsilon_{2} v_{1}}{v_{2}}\left[\bar{E}_{1}(t)-\bar{E}_{1}(0)\right] .
$$

The accumulated interfacial charge is thus proportional to the difference between the actual and initial value of $\bar{E}_{1}$.

Next, substitution of Eqs. (10) and (12) into Eq. (13) gives the final value of the stored charge density

$$
q(\infty)=E_{\mathrm{p} 0} \frac{\kappa_{2} \varepsilon_{1}-\kappa_{1} \varepsilon_{2}}{\kappa_{1} v_{2}+\kappa_{2} v_{1}} .
$$

We see that the interfacial charge $q(\infty)$ increases linearly with the applied voltage and is also temperature dependent, because the conductivities $\kappa_{1}$ and $\kappa_{2}$ are dependent on temperature. The sign of difference $\left(\kappa_{2} \varepsilon_{1}-\varepsilon_{2} \kappa_{1}\right)$ determines whether $q(t)$ will be positive or negative. The system acquires the largest charge when the 
conductivities $\kappa_{1}$ and $\kappa_{2}$ differ significantly. In limiting case when $\kappa_{1} \ll \kappa_{2}$ Eq. (14) reduces to

$$
q(\infty)=\frac{\varepsilon_{0} \varepsilon_{2}}{v_{2}} E_{\mathrm{p}}
$$

giving an interfacial charge that is independent of the polarization temperature.

Now, we can return to the TSD current of the parallel-plate condenser. In this case the applied voltage $U_{\mathrm{p}}=0$. Thus, Eq. (2) can be written as

$$
v_{1} \bar{E}_{1}+v_{2} \bar{E}_{2}=0 \text {. }
$$

During the TSD measurements the external voltage $U_{\mathrm{p}}$ is switched off at a low temperature $T_{0}$, and the system is heated up, usually at a constant rate $b=\mathrm{d} T / \mathrm{d} t$, i.e.

$$
T=T_{0}+b t .
$$

The current which flows in the external circuit, due to depolarization processes, is recorded as a function of temperature $T$. Introduction of a new variable $T$ into Eqs. (6) and (15) yields the system

$$
\begin{aligned}
& \kappa_{1}(T) \bar{E}_{1}(T)+b \varepsilon_{0} \varepsilon_{1} \frac{\mathrm{d} \bar{E}_{1}(T)}{\mathrm{d} T}=\kappa_{2}(T) \bar{E}_{2}(T)+b \varepsilon_{0} \varepsilon_{2} \frac{\mathrm{d} \bar{E}_{2}(T)}{\mathrm{d} T}, \\
& v_{1} \bar{E}_{1}(T)+v_{2} \bar{E}_{2}(T)=0 .
\end{aligned}
$$

By eliminating $\bar{E}_{2}(T)$ from these equations we obtain that

$$
\frac{\mathrm{d} \bar{E}_{1}(T)}{\mathrm{d} T}=-\frac{1}{b \tau(T)} \bar{E}_{1}(T)
$$

where $\tau$ is given by Eq. (8). The solution of differential Eq. (17) is

$$
\bar{E}_{1}(T)=\bar{E}_{10}\left(T_{\mathrm{p}}\right) \exp \left[-\int_{T_{0}}^{T} \frac{\mathrm{d} T}{b \tau(T)}\right] .
$$

The initial value $\bar{E}_{10}=\left.\bar{E}_{1}(T)\right|_{T=T_{0}}$ is determined by the interfacial charge accumulated during the polarization of the system. To calculate this constant we can use Eqs. (16), (5) and (14). Consequently, we obtain that

$$
\bar{E}_{10}=E_{\mathrm{p}} \frac{\kappa_{2} \varepsilon_{1}-\kappa_{1} \varepsilon_{2}}{\kappa_{1} v_{2}+\kappa_{2} v_{1}} \frac{v_{2}}{\varepsilon_{1} v_{2}+\varepsilon_{2} v_{1}} .
$$

For the TSD current density $J(T)$ we have

$$
J(T)=\kappa_{1}(T) \bar{E}_{1}(T)+b \varepsilon_{0} \varepsilon_{1} \frac{\mathrm{d} \bar{E}_{1}(T)}{\mathrm{d} T} .
$$

By substituting into this equation the function $\mathrm{d} \bar{E}_{1}(T) / \mathrm{d} T$ from Eq. (17)' and introducing $\tau$ from Eq. (8) we obtain

$$
J(T)=\bar{E}_{1}(T) v_{1} \frac{\kappa_{1}(T) \varepsilon_{2}-\kappa_{2}(T) \varepsilon_{1}}{\varepsilon_{1} v_{2}+\varepsilon_{2} v_{1}} .
$$

Consequently, by taking into account Eqs. (18) and (19), we can write the following expression

$$
J(T)=E_{\mathrm{p}} \text { const }\left[\kappa_{1}(T) \varepsilon_{2}-\kappa_{2}(T) \varepsilon_{1}\right] \exp \left[-\frac{1}{b} \int_{T_{0}}^{T} \frac{\mathrm{d} T^{\prime}}{\tau\left(T^{\prime}\right)}\right],
$$


where

$$
\text { const }=\frac{v_{1} v_{2}}{\left(\varepsilon_{1} v_{2}+v_{1} \varepsilon_{2}\right)^{2}} \frac{\kappa_{2}\left(T_{\mathrm{p}}\right) \varepsilon_{1}-\kappa_{1}\left(T_{\mathrm{p}}\right) \varepsilon_{2}}{\kappa_{2}\left(T_{\mathrm{p}}\right) v_{1}+\kappa_{1}\left(T_{\mathrm{p}}\right) v_{2}}
$$

and

$$
\tau^{-1}=\frac{\kappa_{1}(T) v_{2}+\kappa_{2}(T) v_{1}}{\varepsilon_{0}\left(\varepsilon_{1} v_{2}+v_{1} \varepsilon_{2}\right)}
$$

This equation shows that TSD current depends on two varying quantities: $\kappa_{1}$ and $\kappa_{2}$. The shape and location of the TSD current peaks are dependent on the dielectric properties and volume fractions of both materials and on the value of the charge accumulated at the polarization temperature $\mathcal{T}_{\mathrm{p}}$. Equation (20) was obtained to describe TSD currents of a parallel-plate condenser filled with the two-component heterogeneous solid. This equation may be used also to describe the TSD currents of two-layer parallel-plate condenser because in this case $v_{1}=S d_{1}$ and $v_{2}=S d_{2}$ (where $S$ - the surface and $d_{1}$ and $d_{2}$ - the thicknesses of layers), and Eq. (20) reduces to those obtained by Harašta and Thurzo [13] and by van Turnhout [4].

\section{References}

[1] L.K.H. van Beek, in: Progress in Dielectric, Ed. J.B. Birks, Vol. 7, Heywood Books, London 1967, p. 69.

[2] J. Volger, in: Progress in Semiconductors, Ed. A.F. Gibson, Vol. 4, Heywood and Comp. Ltd., London 1960, p. 205.

[3] A.K. Jonscher, Dielectric Relaxation in Solids, Chelsea Dielectric Press, London 1963.

[4] J. van Turnhout, Thermally Stimulated Discharge of Polymer Electrets, Elsevier, Amsterdam 1975, Chapt. 6.

[5] J. Vanderschueren, J. Gasiot, in: Thermally Stimulated Relaxation in Solids, in series Topics in Applied Physics, Ed. P. Braunlich, Vol. 37, Springer-Verlag, Berlin 1979, Chapt. 4.

[6] J. van Turnhout, in: Electrets, in series Topics in Applied Physics, Ed. C.M. Sessler, Vol. 33, Springer-Verlag, Berlin 1980, Chapt. 3.

[7] B. Hilczer, J. Małecki, Electrets, Elsevier, Amsterdam 1986, Chapt. 6.2.

[8] Yu.A. Gorokhovatski, Osnovy Termodepolarizationnovo Analiza, Moskwa, Nauka 1981 (in Russian).

[9] R. Chen, Y. Kirsh, Analysis of Thermally Stimulated Processes, Pergamon Press, Oxford 1981, Chapts. 1.3, 1.5, 1.9b, and 3 .

[10] D.M. Smyth (Ed.), Thermal and Photostimulated Currents in Insulators, Electrochem. Soc. Inc., New Jersey 1976.

[11] B. Gross, J. Polym. Sci., Polym. Phys. Ed. 10, 1941 (1971).

[12] B. Gross, J. Electrachem. Soc. 119, 855 (1972).

[13] V. Harašta, J. Thurzo, Fyz. Casop. 20, 148 (1970). 